Boise State University

ScholarWorks

$1-1-2002$

Depth Characterization of Shallow Aquifers with Seismic Reflection, Part I-The Failure of NMO Velocity Analysis and Quantitative Error Prediction

John H. Bradford

Boise State University 


\title{
Depth characterization of shallow aquifers with seismic reflection, Part I-The failure of NMO velocity analysis and quantitative error prediction
}

\author{
John H. Bradford*
}

\begin{abstract}
As seismic reflection data become more prevalent as input for quantitative environmental and engineering studies, there is a growing need to assess and improve the accuracy of reflection processing methodologies. It is common for compressional-wave velocities to increase by a factor of four or more where shallow, unconsolidated sediments change from a dry or partially watersaturated regime to full saturation. While this degree of velocity contrast is rare in conventional seismology, it is a common scenario in shallow environments and leads to significant problems when trying to record and interpret reflections within about the first $30 \mathrm{~m}$ below the water table. The problem is compounded in shallow reflection studies where problems primarily associated
\end{abstract}

with surface-related noise limit the range of offsets we can use to record reflected energy. For offset-to-depth ratios typically required to record reflections originating in this zone, the assumptions of NMO velocity analysis are violated, leading to very large errors in depth and layer thickness estimates if the Dix equation is assumed valid. For a broad range of velocity profiles, saturated layer thickness will be overestimated by a minimum of $10 \%$ if the boundary of interest is $<30 \mathrm{~m}$ below the water table. The error increases rapidly as the boundary shallows and can be very large $(>100 \%)$ if the saturated layer is $<10 \mathrm{~m}$ thick. This degree of error has a significant and negative impact if quantitative interpretations of aquifer geometry are used in aquifer evaluation such as predictive groundwater flow modeling or total resource estimates.

\section{INTRODUCTION}

As interpreted shallow reflection data become more prevalent as input for quantitative groundwater and engineering studies, it is necessary to evaluate our ability to predict depth and layer thickness accurately based on conventional processing methodologies. Typically, multioffset reflection data are processed by applying an NMO correction based on a stacking velocity $\left(v_{s t k}\right)$ profile that moves all energy to its zero-offset equivalent arrival time $\left(t_{0}\right)$. Traces are then summed in common midpoint (CMP) gathers to produce a stacked section. The inherent NMO assumption is $v_{s t k} \approx v_{r m s}$, where $v_{r m s}$ is the rms velocity. When this approximation is valid, we can extract a depth profile based on the Dix equation (Dix, 1955). While the NMO assumption is often valid above the saturated zone, it breaks down across the water table because of the large velocity contrast that typically exists across this boundary. It is common for the compressional-wave velocity to increase by a factor of four or more where shallow, unconsolidated sedi- ments change from a dry or partially saturated regime to full saturation. I refer to this transitional boundary as the water table $(W)$, recognizing that full water saturation actually occurs slightly above the water table as a result of capillary forces. Although this severe velocity gradient has received much attention in the literature (Bachrach and Nur, 1998; Bachrach et al., 1998; Miller and Xia, 1998), there has been no quantitative discussion of the errors resulting from the NMO approximation under these conditions.

I first discuss problems with coherent noise and signal resolution, and how these problems limit the range of offsets we can use for velocity analysis. The remainder of the discussion is focused on a detailed and quantitative analysis of the errors related to conventional NMO velocity analysis. The general arguments and equations are well known but have received little attention in the shallow reflection literature. My intent is to summarize this information in the context of the special conditions that arise in the shallow environment and to present specific relevant calculations. Although the discussion is focused

Manuscript received by the Editor December 21, 1998; revised manuscript received November 21, 2000.

${ }^{*}$ Formerly Rice University, Department of Geology and Geophysics, Houston, Texas 77005; presently Boise State University, CGISS, 1910 University Drive, Boise, Idaho 83725. E-mail: johnb@cgiss.boisestate.edu.

(C) 2002 Society of Exploration Geophysicists. All rights reserved. 
on the transition from a dry or partially saturated regime to fully saturated regime, the arguments apply to any large vertical velocity gradient such as the transition from unconsolidated sediments to bedrock or a sediment-salt interface.

\section{PROBLEMS WITH RECORDING AND PROCESSING REFLECTIONS ORIGINATING BELOW THE WATER TABLE}

It is well known that compressional-wave velocities in dry, unconsolidated sediments are relatively low and decrease slightly as the pore space becomes partially water saturated. When water saturation levels reach $95-99 \%$, the velocity increases abruptly to the velocity at full saturation (Gassman, 1951; Castagna et al., 1993; Bachrach and Nur, 1998; Bachrach et al., 1998). Fully saturated sediment velocities are almost exclusively greater than or equal to water velocity. Only in the case of very high porosity $(\sim 65 \%)$ have saturated velocities less than water velocity been observed (Brandt, 1960). It is common for dry or partially saturated unconsolidated sediments to have compressional-wave velocities well below $500 \mathrm{~m} / \mathrm{s}$, so that a velocity increase of a factor of four or more is typical across the water table (Figure 1). This severe velocity gradient is rarely observed in conventional exploration-scale surveys and violates many of the assumptions of standard NMO velocity analysis schemes. Even though the increase in water saturation from $0-100 \%$ may be a gradual function of depth, the large velocity increase occurs over a very small range of water saturation levels (Figure 1), so we expect the velocity change across the water table to be a sharp boundary relative to the seismic wavelength. This expectation is supported by field observations. Bachrach et al. (1998) present an example of sand with very high volumetric water concentrations (near full saturation) that has a velocity very close to the velocity of the dry sand and much lower $(\sim 1 / 14)$ than the velocity of the saturated sand. The sharp velocity contrast leads to several significant problems; the following three can have a significant impact on velocity analysis:

1) additional sources of coherent noise,

2) geometric loss of resolution, and

3) departure from NMO.

The first two categories can significantly alter the range of offsets available for velocity analysis and are general problems

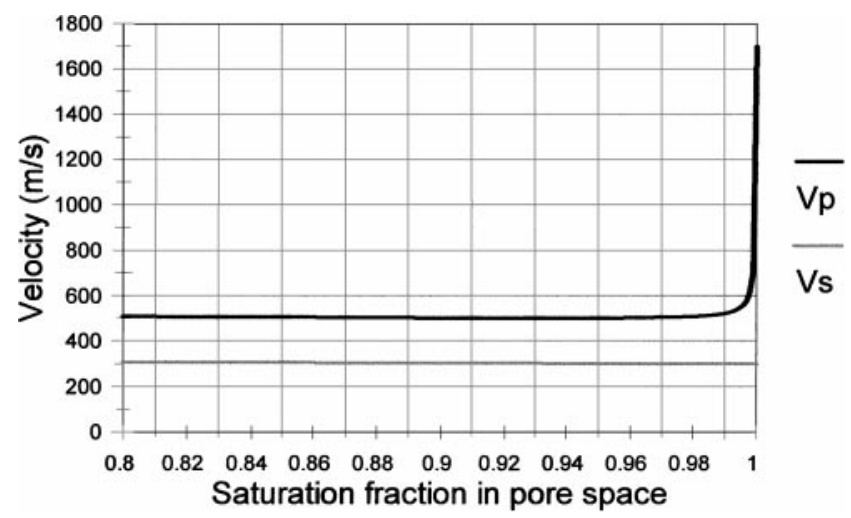

FIG. 1. $P$ - and $S$-velocities for a quartz sand as a function of water saturation. The curves are calculated with Gassman's equations, assuming a burial depth of $10 \mathrm{~m}$. that should be considered in any processing scheme. The third category poses problems specific to extracting interval velocities based on conventional NMO velocity analysis and is significantly affected by the first the two. I briefly discuss the first two categories, focusing on how they affect the range of offsets available for velocity analysis. The remainder of the discussion is devoted to errors that can result from conventional NMO velocity analysis.

\section{Coherent noise}

The air wave and ground roll are strong sources of coherent noise that are often problematic in shallow reflection surveys. Depending on the depth to the water table, heterogeneity of the vadose zone, and the velocity contrast across the water table, additional sources of high-amplitude, low-velocity coherent noise can be generated which interfere with deeper, primary reflections. This includes guided waves trapped between the surface and the saturated zone (Robertsson et al., 1996), interbed multiples where significant impedance contrasts exist within the vadose zone, and converted modes generated at the water table (Figures 2 and 3 ). Each of these noise signals travels at velocities lower than the highest $P$-wave velocity above the saturated zone, so that for $v_{s a t} / v_{d r y} \gg 1$, interference with

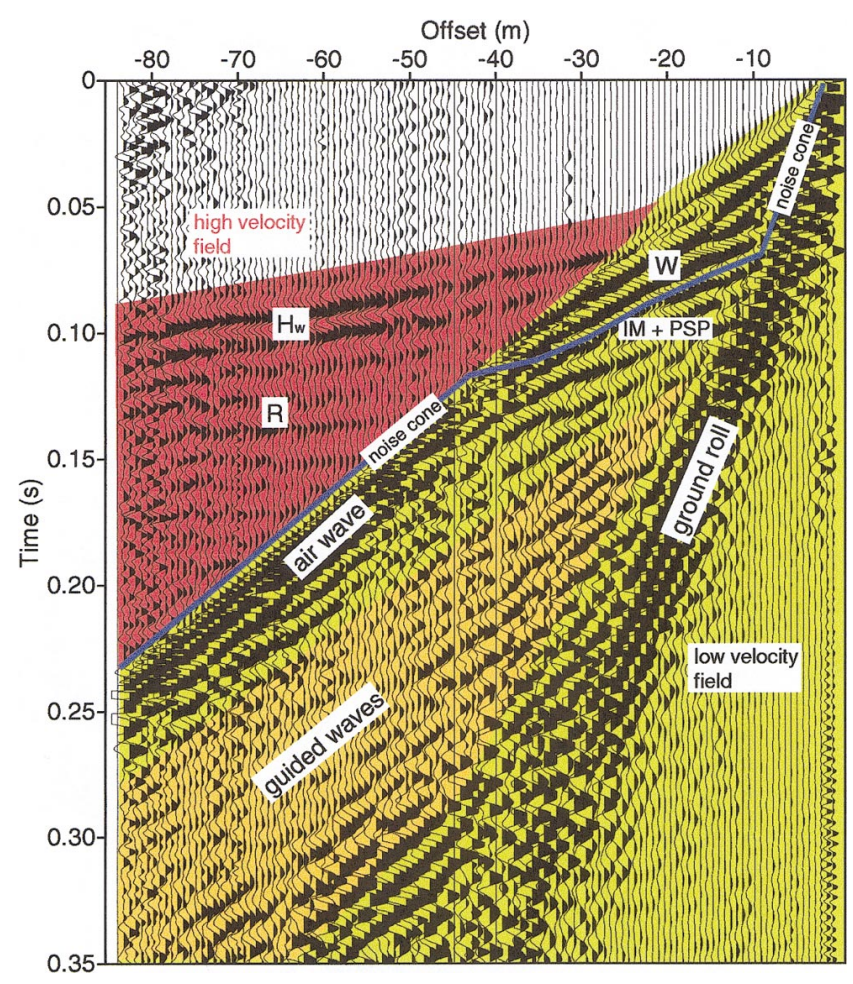

FIG. 2. Shot record illustrating features commonly observed in shallow seismic data. $W=$ water table reflection, $H_{w}=$ water table refraction, $R=$ deeper reflections. The interpretation of an interbed multiple (IM) and PSP-converted mode is based on elastic modeling of the known stratigraphy at the site. The PSP mode is compressional to the water table, converted to shear on reflection, then converted to compressional upon upward transmission through a shallower layer. Yellow indicates the low-velocity field; red indicates the high-velocity field. At $W$ and above, the noise cone is defined by ground roll. 
deeper reflections occurs in the near-offset regime. Interbed multiples, generated above the saturated zone, pose an exceptional problem since they can have stacking velocities consistent with an increase in velocity with depth, leading to misinterpretation. The traveltime curve and arrival time of these events, at near-zero offset, may not be substantially different from primary reflections (although they are easily identified with sufficient offset). Velocity filtering is ineffective at separating this energy at near offset.

Baker et al. (1998) discuss a region defined as the noise cone, within which primary reflected energy is masked by slow, highamplitude coherent noise (Figure 2). The noise cone is contained within the $t-x$ boundary defined by moveout at the noise cone velocity $\left(v_{n c}\right)$. The noise cone velocity depends on site conditions and may be defined by the direct wave, a guided-wave mode, a ground-roll mode, or the direct air wave. Below the water table $(W)$ reflection, additional noise is generated and primary reflected energy is relatively weak because of transmission losses at the water table boundary. Therefore, the noise cone may be different above and below the water table with $\left.v_{n c}\right|_{t<t w} \leq\left. v_{n c}\right|_{t>t w}$, where $t_{W}$ is the zero-offset arrival time of $W$ (Figure 2). In many cases, coherent noise within this region has amplitudes that are one or more orders of magnitude greater than the primary reflections, may be in the same frequency band (depending on the type of noise), and is often spatially aliased. It is often difficult or impossible to remove this noise effectively, and we can extract primary reflected energy only at offsets greater than those defined by $v_{n c}$-particularly when the target reflectors are only a few meters $(<30 \mathrm{~m})$ below the water table.

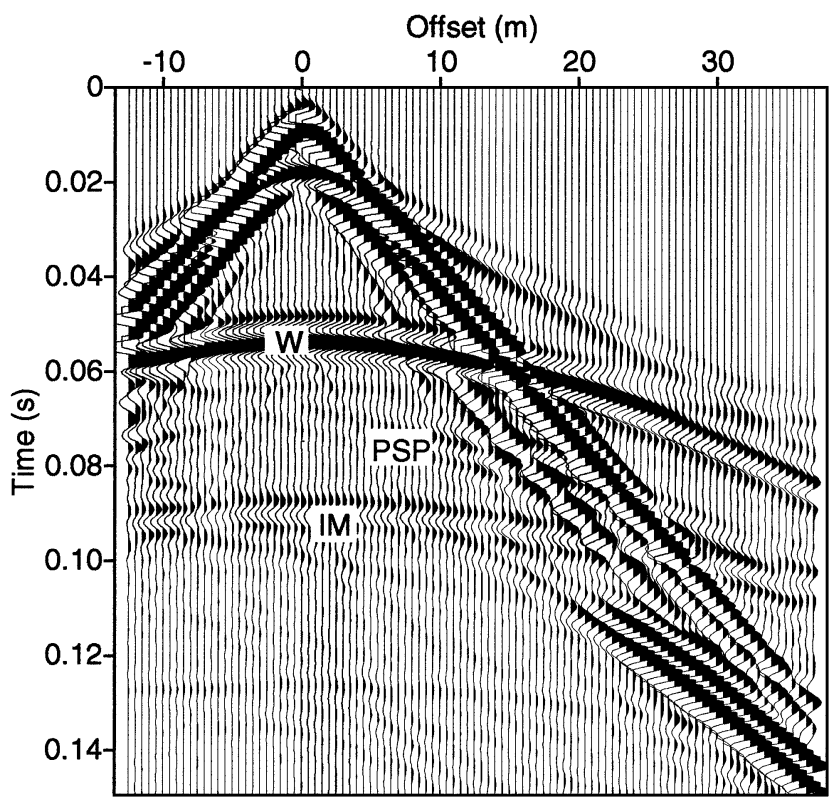

FIG. 3. Viscoelastic synthetic seismogram based on a velocity model derived from traveltime inversion of picks from Figure 3 (Bradford, 1998). W = water table reflection, $\mathrm{PSP}=\mathrm{PSP}$ converted mode (wave is compressional to the watertable, converted to shear on reflection, then converted to compressional upon upward transmission through a shallower layer), $\mathrm{IM}=$ interbed multiple.

\section{Geometric loss of resolution}

Vertical resolution is controlled by the value of the vertical wavenumber. As the vertical component of the wavenumber decreases, resolution potential decreases. As offsets increase, the lateral component of motion increases; therefore, the vertical wavenumber decreases, resulting in decreased vertical resolution (Levin, 1998). This means that the theoretical resolution limit of $\lambda / 4$, where $\lambda$ is the wavelength, is only valid at zero offset. In data, this is observed as reflector convergence with increasing offset. The rate at which resolution decreases with increasing offset depends strongly on the velocity field through which the wave propagates. As the wave travels across a large positive velocity contrast, the lateral component of motion becomes large at relatively small offsets because of severe ray bending (Figure 4). This implies that reflectors will converge with the head wave at relatively small offsets.

For a two-layer case, traveltimes for the head wave $\left(t_{h}\right)$ traveling along the top of the second layer and for the reflection from the base of the second layer $\left(t_{r}\right)$ are given by

$$
t_{h}=\frac{2 z_{1}}{v_{1} \cos \left[\sin ^{-1}\left(\frac{v_{1}}{v_{2}}\right)\right]}+\frac{x-2 z_{1} \tan \left[\sin ^{-1}\left(\frac{v_{1}}{v_{2}}\right)\right]}{v_{2}},
$$
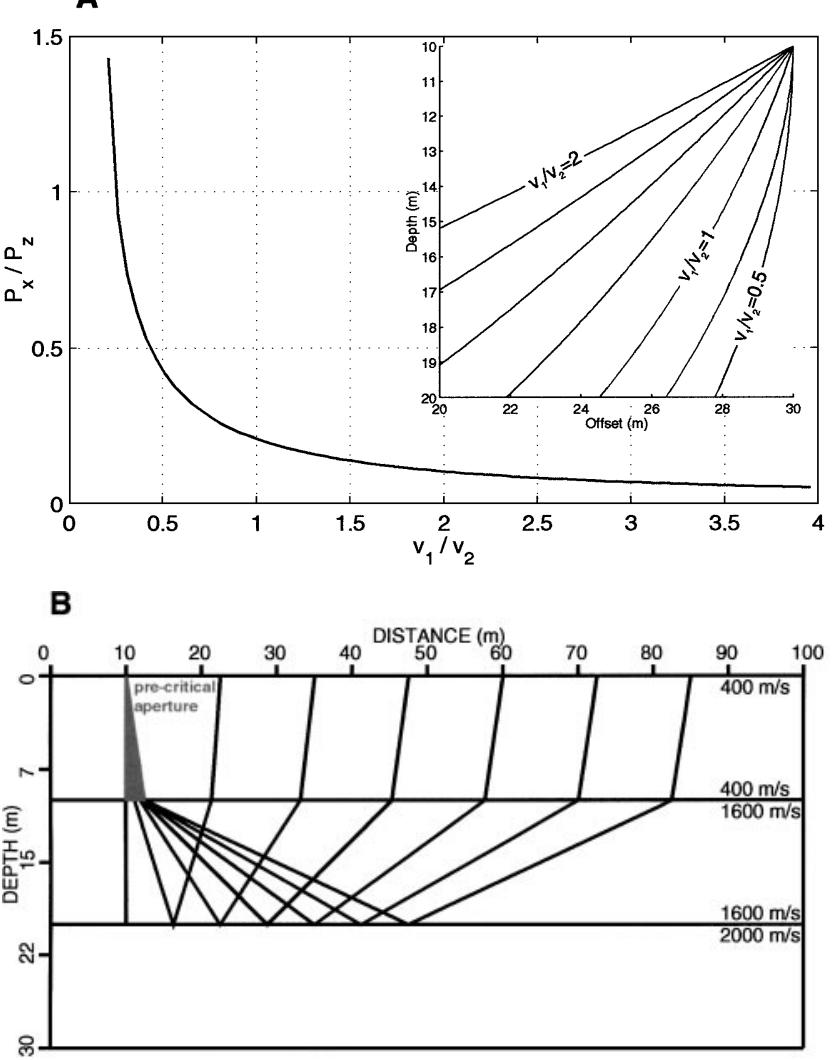

FIG. 4. (a) Ratio of lateral $\left(P_{x}\right)$ to vertical $\left(P_{z}\right)$ components of compressional-wave motion for the transmitted wave versus velocity contrast at $\theta_{1}=14^{\circ}$. The inset shows wavefronts for a range of velocity contrasts, with the velocity boundary at a depth of $10 \mathrm{~m}$. (b) Ray bending across the water table. All energy reflected from the base of the second layer must pass through the precritical aperture. 


$$
\begin{aligned}
t_{r}= & \frac{2 z_{1}}{v_{1} \cos \left[\sin ^{-1}\left(\frac{v_{1}}{v_{2}} \sin \theta_{2}\right)\right]} \\
& +\frac{x-2 z_{1} \tan \left[\sin ^{-1}\left(\frac{v_{1}}{v_{2}} \sin \theta_{2}\right)\right]}{v_{2} \sin \theta_{2}} .
\end{aligned}
$$

Nomenclature is defined in Figure 5. For $t_{r}$,

$$
\begin{aligned}
\frac{x}{2} & =z_{1} \tan \theta_{1}+z_{2} \tan \theta_{2}, \\
\tan \theta_{2} & =\frac{\left(\frac{x}{2}\right)-z_{1} \tan \theta_{1}}{z_{2}} .
\end{aligned}
$$

From equations (3) and (4) and from Snell's law, we see that as $\theta_{1}$ approaches the critical angle $\left(\theta_{c}\right), \theta_{2} \rightarrow \pi / 2$ and $x \rightarrow \infty$, or as $z_{2} \rightarrow 0, \theta_{2} \rightarrow \pi / 2$. We see from equations (1) and (2) that in either case $t_{r}$ approaches $t_{h}$. More importantly, we also find that $t_{r}$ approaches $t_{h}$ as $v_{1} / v_{2}$ decreases (Figure 6). In the context of the present discussion, the presence of a large velocity contrast across the water table means that energy reflected from within the saturated zone will interfere with the water table refraction at relatively small offsets. This further limits the range of offsets available for velocity analysis. The NMO equation actually predicts divergence of $t_{r}$ and $t_{h}$ (Figure 6). This is merely an indication that the NMO approximation breaks down as the offset-to-depth ratio and/or vertical velocity contrast increases.

To illustrate the effect of head wave convergence, consider an earth model with the water table at a depth of $10 \mathrm{~m}$, vadose zone velocity of $400 \mathrm{~m} / \mathrm{s}$, and saturated velocity of $1600 \mathrm{~m} / \mathrm{s}$. There are four reflecting interfaces $\left(R_{5}, R_{10}, R_{15}, R_{20}\right)$ at $5-\mathrm{m}$ intervals below the water table. Now I compute a synthetic shot gather using a fourth-order, finite-difference acoustic modeling code (Figure 7). The center frequency of the source wavelet is $500 \mathrm{~Hz}$, which is near the upper limit that is currently observed in field studies (Steeples, 1998). This gives a wavelength of $3.2 \mathrm{~m}$ in the saturated zone, corresponding to a theoretical resolution of $0.8 \mathrm{~m}$. From this criterion we would expect to easily resolve the four reflectors below the water table. At zero offset, all reflecting interfaces are clearly resolved. With increasing offset, the reflections begin to converge with the water table refraction and with each other. At an offset of around $10 \mathrm{~m}, R_{5}$ begins to interfere significantly with the head wave, and the two wavelets

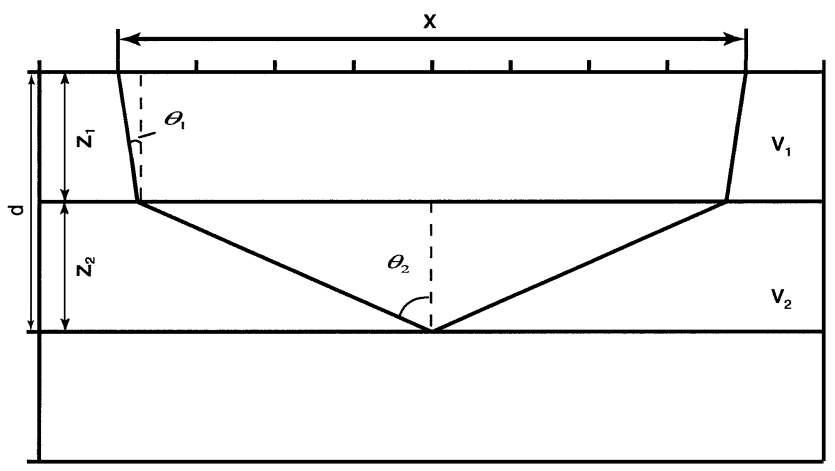

FIG. 5. Ray diagram, indicating parameters used in modeling.

\section{Bradford}

cannot be distinguished at an offset of $25 \mathrm{~m}$. The useable portion of $R_{5}$ lies entirely within the $400-\mathrm{m} / \mathrm{s}$ direct arrival cone. If we were unable to effectively remove coherent noise within this region, we would not observe any portion of $R_{5}$. We can distinguish $R_{10}$ to an offset of about $40 \mathrm{~m}, R_{15}$ to $50 \mathrm{~m}$, and $R_{20}$ to the maximum offset of $60 \mathrm{~m}$.

\section{Departure from NMO}

Considering the ground to be composed of uniform horizontal layers, the traveltime $t_{r}$ for a reflection from the base of the $N$ th layer and offset $x$ are related by the parametric equations (Al-Chalabi, 1973)

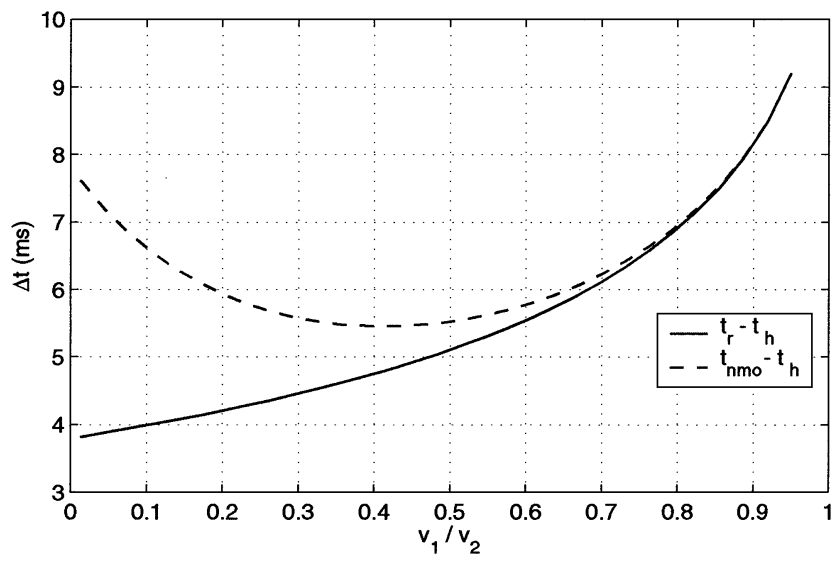

FIG. 6. Head wave convergence at $x=30 \mathrm{~m}$ as a function of velocity contrast for a reflection $10 \mathrm{~m}$ below the water table, where $t_{h}$ is the head wave traveltime, $t_{r}$ is the correct reflection traveltime, and $t_{n m o}$ is the NMO approximation $\left(z_{1}=10 \mathrm{~m}\right)$.

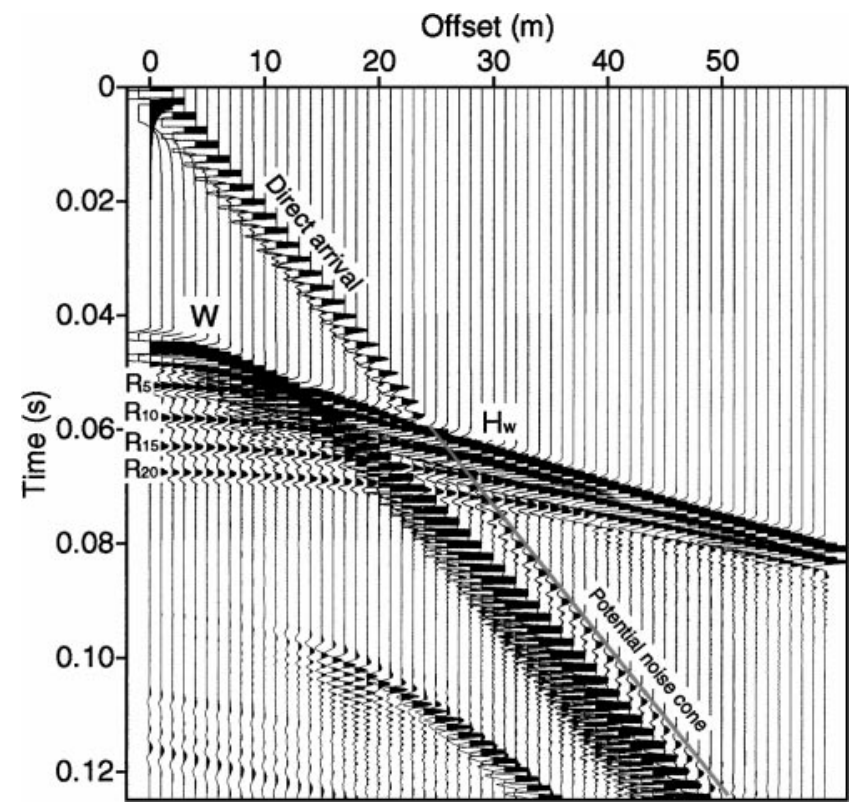

FIG. 7. Acoustic seismogram for a model with four reflectors $\left(R_{5}-R_{20}\right)$ spaced at $5-\mathrm{m}$ intervals below the water table $(W)$, which is $10 \mathrm{~m}$ deep. Head wave and reflector convergence decrease resolving power at far offsets. 


$$
\begin{aligned}
t_{r} & =2 \sum_{k=1}^{N}\left(\frac{z_{k}}{v_{k}}\right)\left(1-p^{2} v_{k}^{2}\right)^{1 / 2}, \\
x & =2 p \sum_{k=1}^{N}\left(\frac{z_{k}}{v_{k}}\right)\left(1-p^{2} v_{k}^{2}\right)^{1 / 2},
\end{aligned}
$$

where $v_{k}$ and $z_{k}$ are the velocity and thickness of the $k$ th layer and $p$ is the ray parameter. These equations cannot be solved in closed form. To obtain a direct $t-x$ relationship, Taner and Koehler (1969) derived the traveltime curve in terms of an infinite series with the following form:

$$
t_{r}^{2}=C_{1}+C_{2} x^{2}+C_{3} x^{4}+\cdots+C_{j} x^{2 j-2}+\cdots .
$$

In standard NMO velocity analysis, it is assumed that the twoterm truncation of this series is a reasonable approximation to the reflection traveltime given by

$$
t_{r}^{2}=t_{N}^{2}+\frac{1}{v_{r m s}^{2}} x^{2}
$$

where $t_{N}$ is zero-offset traveltime and $v_{r m s}$ is rms velocity at the base of the layer of interest where

$$
\left(v_{r m s}=\left(\frac{1}{t_{N}} \sum_{k=1}^{N} v_{k}^{2} t_{k}\right)^{1 / 2}\right) .
$$

In practice, the stacking velocity $v_{s t k}$ and $t_{N}$ are found by fitting the $t^{2}-x^{2}$ reflection traveltime curve, measured from the data, with equation (8). Then $1 / v_{s t k}^{2}$ is the slope of the curve and the intercept is $t_{N}^{2}$. In conventional processing we assume that $v_{s t k} \approx v_{r m s}$. Interval velocity $v_{N}$ is then typically estimated using Dix inversion, given by (Dix, 1955)

$$
v_{N}^{2} \approx \frac{v_{s t k(N)}^{2} t_{N}-v_{s t k(N-1)}^{2} t_{N-1}}{t_{N}-t_{N-1}} .
$$

Al Chalabi (1974) defines bias ( $B)$ as the error in the approximation $v_{s t k} \approx v_{r m s}$ given by

$$
B=v_{s t k}-v_{r m s} .
$$

He shows that $B$ is always greater than or equal to zero and increases as the minimum or maximum offset increases. Al Chalabi also shows that $B$ increases with increasing vertical velocity heterogeneity and can be quite large, even at relatively small offsets. Considering the two-layer case, the bias increases with increasing velocity contrast, regardless of whether the contrast is positive or negative (Figure 8 ). For a velocity contrast between layers 1 and 2, $v_{s t k(1)}=v_{r m s(1)}$ and $v_{s t k(2)}>v_{r m s(2)}$. It is obvious from equation (9) that $v_{2}$ will be overestimated.

Consider how velocity errors affect depth and layer thickness estimates. Two sources of error result from the assumption that $v_{s t k}=v_{r m s}$. The first is overestimating the interval velocity, as discussed above; the second is overestimating $t_{N}$. As shown below, these two sources of error combine constructively to increase depth and layer thickness errors. First, assume that a least-squares fit to the traveltime curve is a good approximation to the velocity analysis procedure used when estimating $v_{s t k}$ from field data. Next, consider the two-layer case with $v_{1}=$ $400 \mathrm{~m} / \mathrm{s}, v_{2}=1600 \mathrm{~m} / \mathrm{s}, z_{1}=z_{2}=10 \mathrm{~m}$, and the target is the base of the second layer. Near zero offset, the two-term approximation to equation (7) provides a good estimate of the actual traveltime; but as offsets increase, the curves diverge significantly (Figure 9a). Additionally, least-squares fitting of the true traveltime curve overpredicts $t_{N}$. Recall that in many cases, reflection data cannot be extracted inside the noise cone. Assuming the direct arrival defines the noise cone, we are limited to using offsets $>30 \mathrm{~m}$ for the reflector from the base of the second layer. Calculating $v_{s t k}$ from the larger offset range increases $B$ and increases the amount that $t_{N}$ is overpredicted (Figure 9b). This leads to significantly larger depth and layer thickness errors.

For the two-layer case, stacking velocity for a reflection from the base of the first layer is given by $v_{s t k(1)}=v_{1}$; then $z_{1}=1 / 2 v_{s t k(1)} t_{1}$. Layer thickness estimate and the corresponding error for the second layer are given by

$$
\begin{aligned}
z_{2} & =\frac{1}{2}\left[v_{2}\left(t_{2}-t_{1}\right)\right], \\
\delta z_{2} & =\frac{1}{2}\left[\delta v_{2}\left(t_{2}-t_{1}\right)+v_{2}\left(\delta t_{2}\right)\right] .
\end{aligned}
$$

The depth estimate and corresponding error are given by

$$
\begin{aligned}
d_{2} & =z_{1}+z_{2}, \\
\delta d_{2} & =\delta z_{2},
\end{aligned}
$$

assuming $\delta t_{1}=0$ and $\delta z_{1}=0$. We see that in the two-layer case, the contributions from $\delta v_{2}$ and $\delta t_{2}$ add constructively since both are positive.

The error is compounded if we base the depth estimate, $d_{2}$, only on the stacking velocity of the reflection from the base of layer 2. This is the case if we use the optimum window technique (Hunter et al., 1984), the base of layer 2 is the target, and the interval velocity in layer 1 is not measured directly since near-offset information is not recorded. In this case it is often assumed that $v_{s t k(2)} \approx v_{a(2)}$, where $v_{a(N)}$ is the time average velocity to the base of layer $N$. The estimated depth to the base of layer 2 , or $d_{2}^{\prime}$, is then calculated by

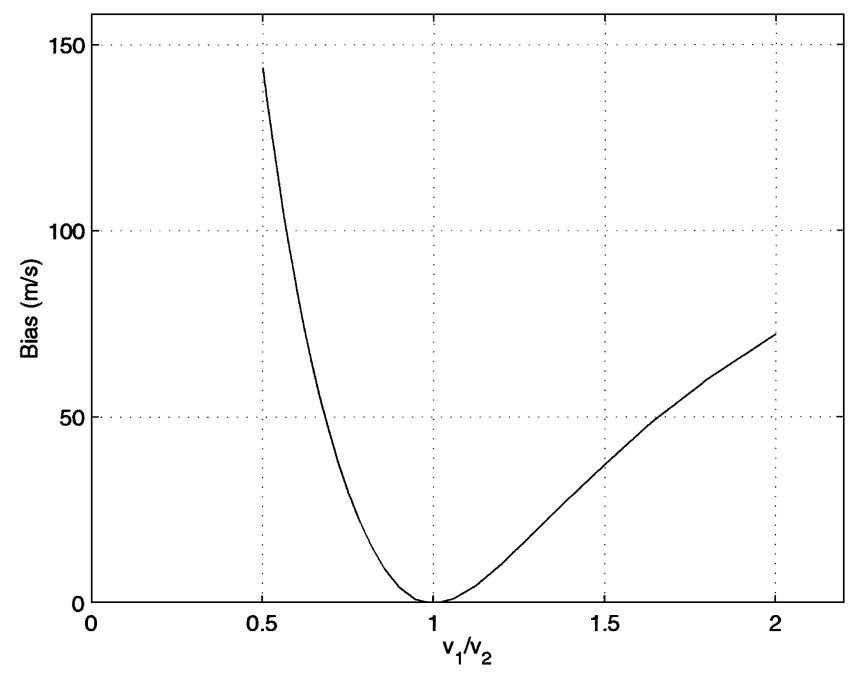

FIG. 8. Velocity bias $\left(v_{s t k}-v_{r m s}\right)$ versus velocity contrast. Bias is computed for a reflection from the base of the second layer with $z_{1}=z_{2}=10 \mathrm{~m}, v_{1}$ held fixed at $900 \mathrm{~m} / \mathrm{s}$, and $v_{2}$ varying from $450-1800 \mathrm{~m} / \mathrm{s}$. Bias is always greater than or equal to zero, regardless of the sign of the velocity contrast. 


$$
d_{2}^{\prime} \approx \frac{1}{2} v_{s t k(2)} t_{2}
$$

If the material above the reflector is highly heterogeneous, this is a very poor approximation. When $v_{2} \gg v_{1}, v_{s t k(2)}$ approaches $v_{2}$ and $v_{2} \gg v_{a(2)}$. The error in the depth estimate becomes

$$
\delta d_{2}^{\prime}=\frac{1}{2}\left[\delta v_{s t k(2)}\left(t_{2}\right)+v_{s t k(2)}\left(\delta t_{2}\right)\right],
$$

where $\delta v_{s t k(2)}=v_{s t k(2)}-v_{a(2)}$. Ignoring $v_{s t k(1)}$ in the computation introduces an additional error term in the depth estimate, given by

$$
d_{2}^{\prime}-d_{2}=\delta d_{2}^{\prime}-\delta d_{2}
$$

At infinite offset, $v_{s t k(2)} \approx v_{2}$, so we can calculate the left side of equation (17) from equations (11), (13), and (15), giving $d_{2}^{\prime}-d_{2}=1 / 2\left(v_{2}-v_{1}\right) t_{1}$ as the maximum additional error. The minimum error will occur when $v_{s t k(2)}=v_{r m s(2)}$. In this case $\delta v_{s t k(2)}=v_{r m s(2)}-v_{a(2)}, \delta v_{2}=0$. We compute the minimum additional error from the right side of equation (17) using equations (12), (14), and (16), giving $\delta d_{2}^{\prime}-\delta d_{2}=1 / 2\left(v_{r m s(2)}-v_{a(2)}\right) t_{2}$. For a homogeneous medium, $\delta d_{2}^{\prime}-\delta d_{2}=0$; but for a highly heterogeneous medium the error can be quite large, even if we perfectly estimate $v_{r m s(2)}$. In practice we will always overesti-
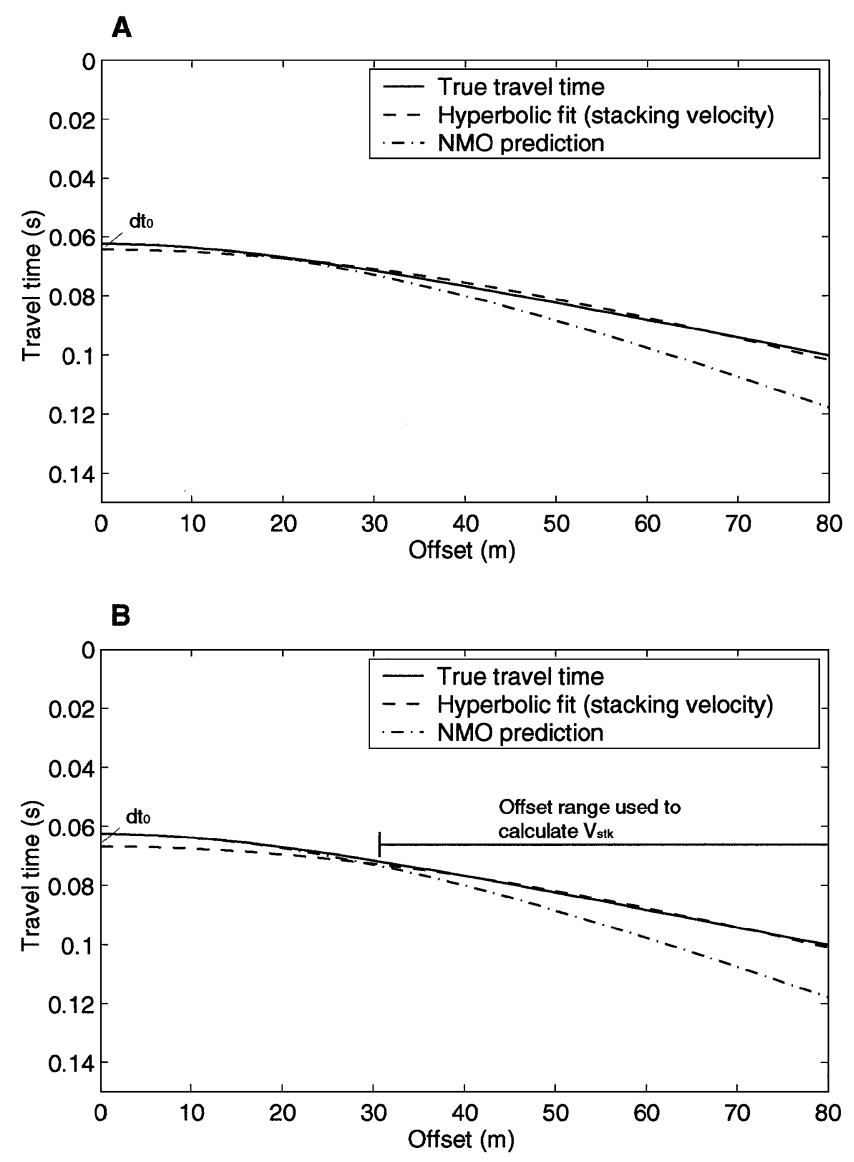

FIG. 9. Reflection traveltime curves (a) using the full offset range to calculate $v_{s t k}$ and (b) using only the far offset range. Using the far offset range only increases $v_{s t k}$ and $\delta t_{o}$. The two-term approximation to the traveltime curve is reasonable at near offsets but provides a poor estimate at far offsets. mate $v_{r m s(2)}$ and $1 / 2\left(v_{r m s(2)}-v_{a(2)}\right) t_{2}<d_{2}^{\prime}-d_{2}<1 / 2\left(v_{2}-v_{1}\right) t_{1}$. As the above analysis indicates, it is very important to consider the low-velocity layer in depth conversion, even if we are not interested in reflections at or above the water table. When the target is $<30 \mathrm{~m}$ below the water-saturated zone, we should acquire sufficient near-offset data to obtain at least a rough estimate of the thickness and average velocity of the vadose zone.

\section{ERROR PREDICTION FOR NMO VELOCITY ANALYSIS}

It is important to quantify the errors discussed up to this point in a practical context. This requires considering all parameters that systematically affect velocity estimates and, ultimately, how these parameters affect the accuracy of depth and layer thickness estimates. Important factors to consider are

1) the thickness of the vadose zone and depth to targets below the water table,

2) the range of offsets available to define $v_{s t k}$, and

3 ) the relative contrast of vadose and saturated-zone velocities.

For the following discussion, consider a two-layer modelthe top layer consisting of a relatively low-velocity material (representing the vadose zone) and the second layer consisting of a high-velocity material (representing the saturated zone). I will calculate and discuss how each of the factors mentioned above affects the estimate of depth to base and the thickness of the second layer. I adopt the following nomenclature:

$R_{1}$-reflection from the base of the low-velocity layer (water table reflection);

$R_{2}$-target reflector within the high-velocity or saturated zone;

$\sigma_{z 2}$-fractional thickness error for layer two $\left(z_{\text {est }(2)}-\right.$ $\left.z_{2}\right) / z_{2}$

$\sigma_{d}$-fractional depth error to the base of layer two $\left(d_{\text {est }(2)}-d_{2}\right) / d_{2}$.

To compute traveltimes for the two-layer case, I use the following procedure. For a given offset, the traveltime $(t)$ for a reflection from the lower boundary of the two-layer model is given by

$$
t_{x}=2\left(\frac{z_{1}}{v_{1} \cos \theta_{1}}+\frac{z_{2}}{v_{2} \cos \theta_{2}}\right) .
$$

Using Snell's law, we write the offset as a function of $\theta_{1}$ :

$$
\frac{x}{2}=z_{1} \tan \theta_{1}+z_{2} \tan \left[\sin ^{-1}\left(\frac{v_{2}}{v_{1}} \sin \theta_{1}\right)\right] .
$$

I solve equation (19) for $\theta_{1}$ numerically using the NewtonRaphson method, with the constraint that $\left|\sin \theta_{1}\right|<v_{1} / v_{2}$. Then $\theta_{2}$ is given by Snell's law. It usually requires less than five iterations to find $\theta_{1}$ to a tolerance of $<1 \%$, even under the most extreme conditions (large velocity contrast, large offset, small $z_{2}$ ).

The stacking velocity for $R_{2}$ is found by fitting equation (8) to the traveltime curve using a least-squares routine. The stacking velocity for $R_{1}$ is $v_{1}$. The interval velocity $v_{2}$ is estimated with the two-layer Dix equation [equation (9)], and $z_{2}$ and $d$ are estimated with equations (11) and (13), respectively.

First consider the effect of varying the thickness of $z_{1}$ and $z_{2}$. In general, $\sigma_{z 2}$ and $\sigma_{d}$ increase with decreasing $z_{2}$ (Figure 10); 
$\sigma_{z 2}$ increases rapidly when $z_{2}<20 \mathrm{~m}$ and becomes very large when $z_{2}<5 \mathrm{~m}$. The dependence of $\sigma_{z 2}$ on $z_{1}$ is relatively weak but does show a local maximum along the $z_{1}$ axis that varies in location with increasing $z_{2}$. When the full stacking velocity field is used to estimate $v_{2}, \sigma_{d}$ is $<20 \%$ for most values of $z_{1}$ and $z_{2}$ but becomes very large when $z_{1}$ or $z_{2}$ is less than about $15 \mathrm{~m}$. When $d$ is estimated based only on $v_{s t k(2)}$ [equation (15)], as may be the case using the optimum window technique, $\sigma_{d}$ is $>30 \%$ over a large range of $z_{1}$ and $z_{2}$. This example illustrates why failing to incorporate the low-velocity zone in depth conversion can lead to very large errors in depth estimate. The problem can be minimized by estimating $v_{r m s(2)}$ using the method of shifting stack (Al-Chalabi, 1974).

Next we consider the effect of varying the offset range used to define $v_{s t k}$. Two factors contribute to the available offset range: (1) maximum offset, which is controlled by spread length or head wave/reflector convergence, and (2) minimum available offset, which is controlled by the shot-to-near-receiver gap or by the prevalence of near-offset coherent noise. As mentioned in the previous section, the bias $B$ increases as either the minimum or maximum offset increases. First consider variation in the maximum offset $\left(x_{\max }\right)$. In general, $\sigma_{z 2}<10 \%$ when the maximum offset-to-depth ratio is $<1$ (Figure 11). But as previously discussed, it is often impossible to extract reflected energy at offsets this small. Also, $\sigma_{z 2}$ is a relatively simple function of $x_{\max }$ and $z_{2}$, increasing as $x_{\max }$ and $z_{2}$ increase, but it is a somewhat more complicated function of $z_{1}$ with a maximum along the $z_{1}$ axis at $z_{1}=z_{2}$.
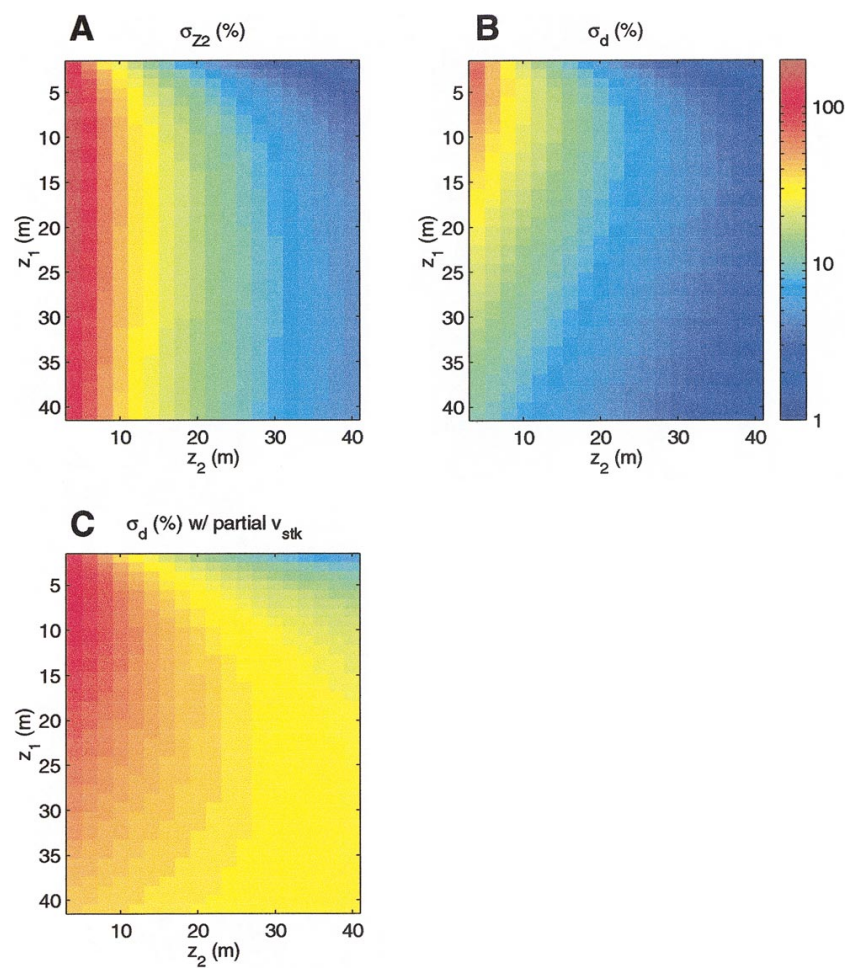

FIG. 10. Error in thickness and depth estimates resulting from NMO velocity analysis and Dix inversion. Error is plotted as a function of $z_{1}$ and $z_{2}$ for a two-layer model with $v_{1}=400 \mathrm{~m} / \mathrm{s}$, $v_{2}=1600 \mathrm{~m} / \mathrm{s}$, and offset range-0-80 m. (a) Layer 2 thickness error $\left(\sigma_{z 2}\right)$. (b) Depth-to-base error $\left(\sigma_{d}\right)$ with full $v_{s t k}(t)$ and Dix inversion. (c) $\sigma_{d}$ with $d$ estimated assuming $v_{s t k(2)} \approx v_{a(2)}$.
Now consider $\sigma_{z 2}$ as a function of minimum offset. For purposes of this discussion, I vary the minimum offset in terms of the noise cone velocity $\left(v_{n c}\right)$. Depending on local site conditions, this could represent ground roll, guided waves, or the direct $P$-wave. In general, $\sigma_{z 2}$ is not strongly dependent on $v_{n c}$ but increases with increasing $v_{n c}$ as expected (Figure 12). For $z_{2}<30 \mathrm{~m}, \sigma_{z 2}>10 \%$ for most values of $v_{n c}$. For the full range of $z_{1}$ used in the calculation, $\sigma_{z 2}>25 \%$ when $z_{2}=10 \mathrm{~m}$.

Finally, consider the variation of $v_{1}$. Typically, there is less relative variation in the saturated velocity than in the vadose zone velocity, and it is $v_{1} / v_{2}$ that controls $\sigma_{z 2}$. By varying only $v_{1}$, we can view the trend in $\sigma_{z 2}$ over a wide range of $v_{1} / v_{2}$; so varying $v_{2}$ would not contribute significantly to our understanding. In general, $\sigma_{z 2}$ decreases with increasing $v_{1}$, which is what we expect since as $v_{1} \rightarrow v_{2}, B \rightarrow 0$ (Figure 13).

\section{RELEVANCE TO GROUNDWATER STUDIES}

Estimating the total volume of water available in an aquifer is probably the simplest calculation relating saturated layer thickness estimates to groundwater studies. Since volume is
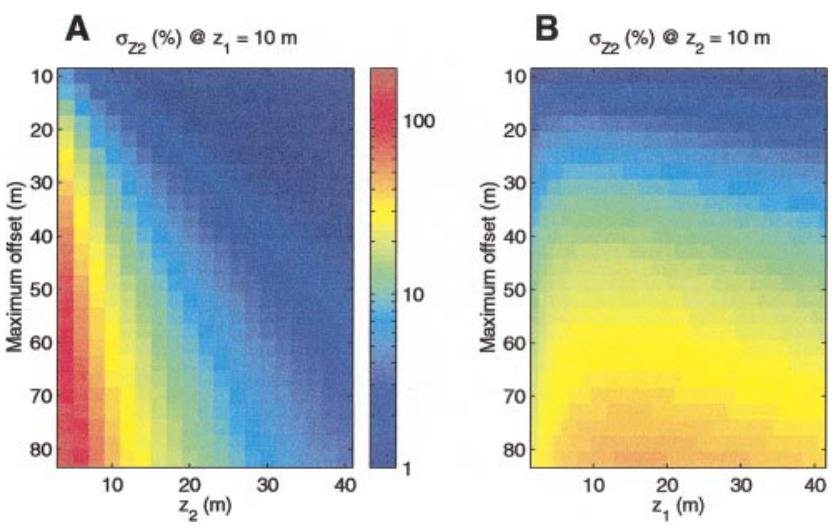

FIG. 11. Layer 2 thickness error $\left(\sigma_{z 2}\right)$ resulting from NMO velocity analysis and Dix inversion. Error is plotted as a function of maximum offset $\left(x_{\max }\right)$ with $v_{1}=400 \mathrm{~m} / \mathrm{s}, v_{2}=1600 \mathrm{~m} / \mathrm{s}$, versus (a) $z_{2}$ and (b) $z_{1}$.
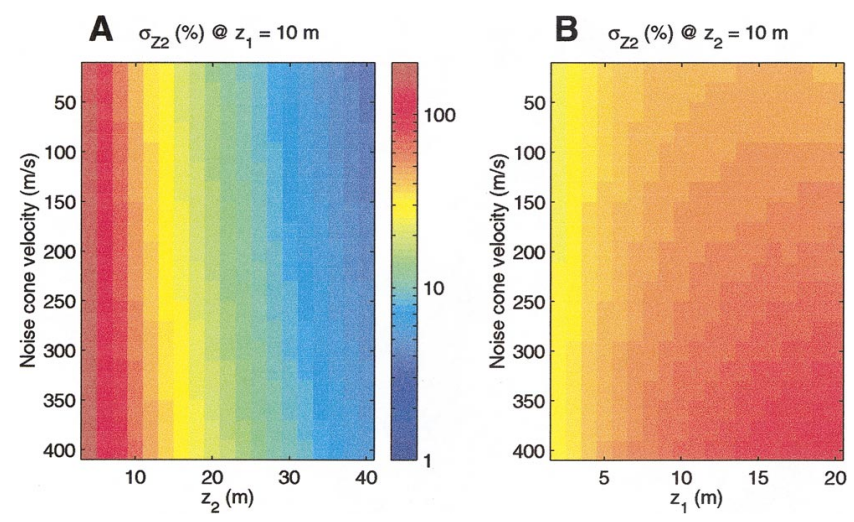

FIG. 12. Layer 2 thickness error $\left(\sigma_{z 2}\right)$ resulting from NMO velocity analysis and Dix inversion. Error is plotted as a function of noise cone velocity $\left(v_{n c}\right)$ versus (a) $z_{2}$ and (b) $z_{1}$ with $v_{1}=$ $400 \mathrm{~m} / \mathrm{s}, v_{2}=1600 \mathrm{~m} / \mathrm{s}$, and $x_{\max }=80 \mathrm{~m}$. 

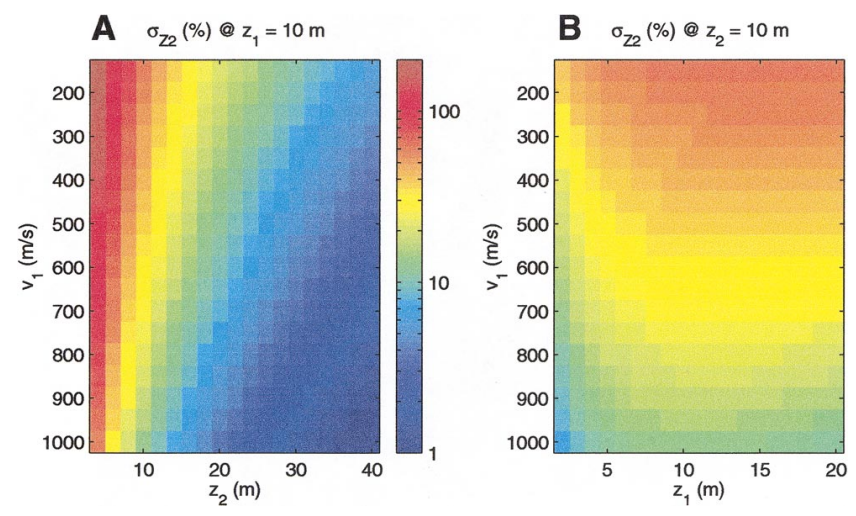

FIG. 13. Layer 2 thickness error $\left(\sigma_{z 2}\right)$ resulting from NMO velocity analysis and Dix inversion. Error is plotted as a function of $v_{1}$ versus (a) $z_{2}$ and (b) $z_{1}$ with $v_{2}=1600 \mathrm{~m} / \mathrm{s}$ and an offset range of $0-80 \mathrm{~m}$.

directly proportional to thickness ( $V=A z_{2}$, where $A$ is surface area), the fractional error in the volumetric estimate $d V / V$ will be identical to the fractional error $\sigma_{z 2}$. This fundamental error propagates through more complex, dynamic calculations. To illustrate this, I present a simple application in well mechanics, based on an example discussed by Bedient et al. (1994). Consider an unconfined, homogeneous aquifer with a prepumping water level of $10.7 \mathrm{~m}$. I want to determine if a specified extraction rate is sustainable for a fully penetrating, pumping well. I do this by estimating the steady-state water level near the well. Bedient et al. (1994) present the equations necessary for the calculation. The calculation is based on a prepumping estimate of saturated thickness $\left(z_{2}\right)$, which could be obtained from a seismic reflection survey. Assume a pumping rate of $3.68 \times 10^{-3} \mathrm{~m}^{3} / \mathrm{s}$ and hydraulic conductivity of $7.07 \times 10^{-5} \mathrm{~m} / \mathrm{s}$. For this aquifer, the pumping rate is not sustainable, since the water level reaches $0 \mathrm{~m}$ at $1 \mathrm{~m}$ from the well (Figure 14). However, if we overestimate the prepumping water level $z_{2}$ by just $10 \%$, the water level is $4.9 \mathrm{~m}$ at $1 \mathrm{~m}$ from the well, indicating that the pumping rate is easily sustainable (Figure 14). This is clearly incorrect. As previously shown, conventional NMO velocity analysis and Dix inversion will, for a broad range of scenarios, result in overestimating the saturated thickness by $>10 \%$. It is necessary to account for this error to predict groundwater flow accurately. In a companion paper, Bradford and Sawyer (2002) discuss several methods for improving interval velocity estimates and image accuracy with a focus on prestack depth migration.

\section{CONCLUSIONS}

As seismic reflection data become more prevalent as input for quantitative environmental and engineering studies, there is a growing need to assess and improve the accuracy of reflection processing methodologies. Prominent surface-related noise often requires that reflections originating within the saturated zone be recorded at relatively large offset-to-depth ratios. Conventional NMO velocity analysis techniques with depth estimates via Dix inversion can result in very large errors in depth and layer thickness estimates for subsurface conditions common in shallow reflection studies.

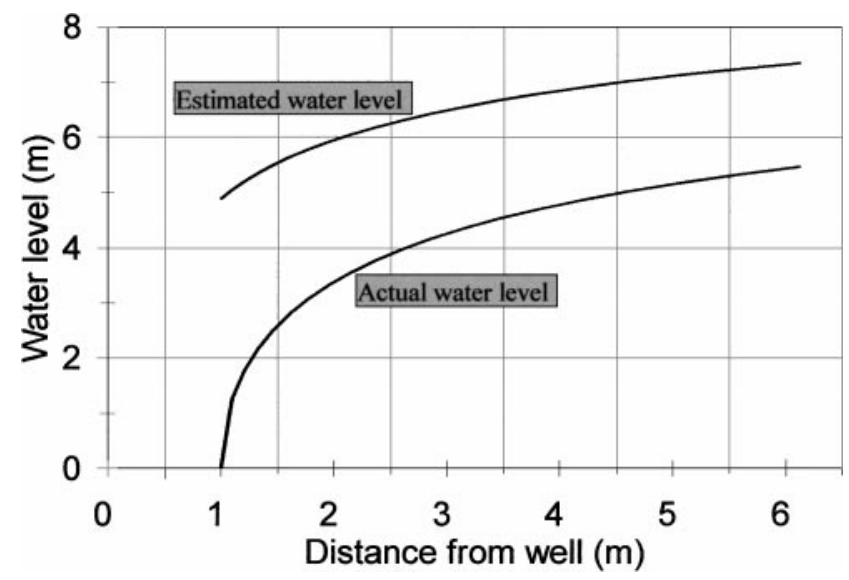

FIG. 14. Steady-state water level near a pumping well and the predicted water level, assuming the prepumping aquifer thickness is overestimated by $10 \%$. Actual prepumping saturated thickness is $10.7 \mathrm{~m}$. The calculation assumes a homogeneous aquifer with hydraulic conductivity of $7.07 \times 10^{-5} \mathrm{~m} / \mathrm{s}$. The well is fully penetrating and is pumping at a rate of $3.16 \times 10^{-3} \mathrm{~m}^{3} / \mathrm{s}$.

In general, fractional error in saturated layer thickness $\left(\sigma_{z 2}\right)$ is a relatively simple function of saturated layer thickness $\left(z_{2}\right)$, decreasing as $z_{2}$ increases. The error is a more complicated function of vadose zone thickness $\left(z_{1}\right)$; with a minimum at $z_{1}=0$, a local maximum that depends on the offset range, ratio $v_{1} / v_{2}$, and $z_{2}$; then a gradual decrease with increasing $z_{1}$. The error increases as maximum and minimum offset increase and decreases as $v_{1} / v_{2}$ approaches 1 . The error can be very large $(>100 \%)$ for a relatively thin saturated layer $(<10 \mathrm{~m})$, but even under a broad range of reasonable scenarios the errors will be $>50 \%$ with typical noise cone velocities of $150-400 \mathrm{~m} / \mathrm{s}$. Errors of just $10 \%$ can have a significant and negative impact on groundwater flow predictions.

Since the error is predictable within the limits of random error and signal resolution, we should devise processing strategies to minimize the uncertainty, thereby improving our prediction of the subsurface. A number of methods treat the problem correctly, including traveltime inversion and migration velocity analysis. While it is impractical to list all applications for which this discussion may apply, it is clear that careful consideration of accuracy problems and the use of appropriate velocity analysis tools can improve the utility of seismic reflection in quantitative groundwater studies.

\section{REFERENCES}

Al-Chalabi, M., 1973, Series approximation in velocity and traveltime computations: Geophys. Prosp., 21, 783-795.

1974, An analysis of stacking, RMS, average, and interval velocities over a horizontally layered ground: Geophys. Prosp., 22, 458475 .

Bachrach, R., and Nur, A., 1998, High-resolution shallow-seismic experiments in sand, Part I-Water table, fluid flow, and saturation: Geophysics, 63, 1225-1233.

Bachrach, R., Dvorkin, J., and Nur, A., 1998, High-resolution shallowseismic experiments in sand, Part II-Velocities in shallow unconsolidated sand: Geophysics, 63, 1234-1240.

Baker, G. S., Steeples, D. W., and Drake, M., 1998, Muting the noise cone in near-surface reflection data: An example from southeastern Kansas: Geophysics, 63, 1332-1338.

Bedient, P. B., Rifai, H. S., and Newell, C. J., 1994, Ground water contamination: Transport and remediation: Prentice-Hall, Inc. 
Bradford, J. H., 1998, Characterizing shallow aquifers with wavepropagation based geophysical techniques: Imaging and attribute analysis: Ph.D. thesis, Rice University.

Bradford, J. H., and Sawyer, D. S., 2002, Depth characterization of shallow aquifers with seismic reflection, Part II-Pre-stack depth migration and field examples: Geophysics, 67, 98-109, this issue.

Brandt, H., 1960, Factors affecting compressional wave velocity in unconsolidated marine sand sediments: J. Acoust. Soc. Am., 32, 171179.

Castagna, J. P., Batzle, M. L., and Kan, T. K., 1993, Rock physics-The link between rock properties and AVO response, in Castagna, J. P., and Backus, M. M., Eds., Offset-dependent reflectivity-Theory and practice of AVO analysis: Soc. Expl. Geophys., Invest. Geophys. 8, 135-171.

Dix, C. H., 1955, Seismic velocities from surface measurements: Geophysics, 20, 180-195.

Gassman, F., 1951, Elastic waves through a packing of spheres: Geophysics, 16, 673-685.
Hunter, J. A., Pullan, S. E., Burns, R. A., Gagne, R. M., and Good, R. S., 1984, Shallow seismic reflection mapping of the overburdenbedrock interface with the engineering seismograph-Some simple techniques: Geophysics, 49, 1381-1385.

Levin, S. A., 1998, Resolution in seismic imaging: Is it all a matter of perspective?: Geophysics, 63, 743-749.

Miller, R. D., and Xia, J., 1998, Large near-surface velocity gradients on shallow seismic reflection data: Geophysics, 63, 13481356.

Robertsson, J. O. A., Holliger, K., Green, A. G., Pugin, A., and De Iaco, R., 1996, Effects of near-surface waveguides on shallow highresolution seismic refraction and reflection data: Geophys. Res. Lett., 23, 495-498.

Steeples, D. W., 1998, Shallow seismic reflection section-Introduction: Geophysics, 63, 1210-1212.

Taner, M. T., and Koehler, F., 1969, Velocity spectra-Digital computer derivations and applications of velocity functions: Geophysics, $\mathbf{3 4}$ $859-881$. 